\title{
Captures
}

Figures, théories et pratiques de l'imaginaire

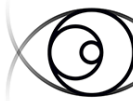

C A P Figures, théories et pratiques de limaginaire

M. Caravan ou l'homme-pendule

L'oppression de la ligne et de la lettre dans En famille de

Maupassant

\section{Émile Bordeleau-Pitre}

Volume 2, Number 2, 2017

Imaginaire de la ligne

URI: https://id.erudit.org/iderudit/1059771ar

DOI: https://doi.org/10.7202/1059771ar

See table of contents

Publisher(s)

Figura, Centre de recherche sur le texte et l'imaginaire

ISSN

2371-1930 (digital)

Explore this journal

Cite this article

Bordeleau-Pitre, É. (2017). M. Caravan ou l'homme-pendule : l'oppression de la ligne et de la lettre dans En famille de Maupassant. Captures, 2(2).

https://doi.org/10.7202/1059771ar
Article abstract

What reading may one make of Guy de Maupassant's "En famille” when heeding Tim Ingold's theory of lines? Whether real or imaginary, the lines in this short story oppose vertical and horizontal tensions. To succeed in the bureaucratic world in which Maupassant's characters live they must live by and master the codes dictated by the straight line, which is something M. Caravan will never be able to achieve.
Tous droits réservés @ Émile Bordeleau-Pitre, 2017

\section{(c) (i) $\Theta$}

This document is protected by copyright law. Use of the services of Érudit (including reproduction) is subject to its terms and conditions, which can be viewed online.

https://apropos.erudit.org/en/users/policy-on-use/
This article is disseminated and preserved by Érudit.

Érudit is a non-profit inter-university consortium of the Université de Montréal, Université Laval, and the Université du Québec à Montréal. Its mission is to promote and disseminate research.

https://www.erudit.org/en/ 
Version enrichie de cet article : http://revuecaptures.org/node/996

\section{Caravan ou l'homme-pendule}

\section{L'oppression de la ligne et de la lettre dans En famille de Maupassant}

\section{Émile Bordeleau-Pitre}

Résumé :

À partir de l'anthropologie comparée de la ligne de Tim Ingold, cet article aborde la nouvelle «En famille » de Guy de Maupassant. Réelles ou imaginaires, les lignes y forment une structure horizontale et verticale où se confrontent des valeurs radicalement opposées. Pour réussir dans le monde bureaucratisé de la nouvelle, il faut connaître et maîtriser les codes que dicte la ligne droite, ce que n'arrivera jamais à faire M. Caravan, personnage principal de la nouvelle.

What reading may one make of Guy de Maupassant's "En famille" when heeding Tim Ingold's theory of lines? Whether real or imaginary, the lines in this short story oppose vertical and horizontal tensions. To succeed in the bureaucratic world in which Maupassant's characters live they must live by and master the codes dictated by the straight line, which is something M. Caravan will never be able to achieve.

Dans Une brève histoire des lignes, Tim Ingold, souhaitant faire ce qu'il qualifie d' " anthropologie comparée de la ligne » (2011: 7), expose l'omniprésence de cette dernière dans nos vies, dans la nature ainsi que dans toute activité humaine : qu'elles existent dans l'espace sous forme de fils (une pelote, un circuit électrique, des cordes de violon, un pont suspendu par exemple) ou qu'elles apparaissent sur une surface sous forme de traces (l'écriture, le dessin, les empreintes laissées sur le sol); qu'elles soient le résultat d'une coupure, d'une fissure ou d'une pliure ou qu'elles soient fantômes comme celles, invisibles, qui forment les constellations ou les frontières nationales, les lignes sont partout, nous dit Ingold. Et dans toute ligne s'inscrit un geste, un mouvement : s'y pencher, c'est tenter de comprendre leur nature, la manière dont elles fonctionnent de même que les conséquences profondes qu'elles ont sur la façon dont nous percevons le réel et agissons sur lui.

Que peut nous dire la ligne de certains textes dans lesquels elle est omniprésente? En famille, nouvelle écrite par Guy de Maupassant et publiée en 1881 dans La Maison Tellier, texte peu commenté par la critique, constitue à cet égard un cas des plus intéressants. Réelles parfois, imaginaires le plus souvent, les lignes forment dans le récit une structure partagée entre horizontalité et verticalité où se confrontent des valeurs et des idéologies radicalement opposées. Et pour réussir dans le monde bureaucratisé mis en scène par En famille, il faut connaître et maîtriser les codes que dictent la ligne droite - ce que n'arrivera jamais à faire M. Caravan, personnage principal de la nouvelle.

Dans un premier temps, nous nous pencherons sur les différentes lignes qui découpent le texte. Horizontales, 
elles représentent l'uniformisation, la monotonie, l'absence de changements; verticales, elles sont les signes de la hiérarchisation, des échelons, des gradations. Souhaitant accéder à un poste supérieur, M. Caravan reste, nous verrons comment, prisonnier d'une horizontalité indépassable.

Cela nous amènera à nous éloigner un peu de la ligne droite pour faire un détour par un deuxième aspect de la raison graphique (Goody, 1979) : la lettre. Le poids de la culture écrite — la littératie — avec les rapports, les comptes et les calculs qui la sous-tendent, impose à ceux qui en font partie certaines habiletés spécifiques et un contrôle du corps - habiletés et contrôle complètement étrangers à M. Caravan. Opprimé par la ligne, ce dernier le sera également par la lettre.

\section{Caravan, l'homme-pendule}

Avant de nous pencher sur ce qu'elle suppose et implique, il nous faut d'abord nous arrêter sur la place qu'occupe, dans les faits, la ligne droite au sein d'En famille. Comment se matérialise-t-elle véritablement? Nous avons parlé d'horizontalité et de verticalité de la ligne : quelles caractéristiques, quelles propriétés les définissent et les différencient, et comment se manifestent-elles dans le texte?

C'est dès la première phrase qu'apparaît la ligne dans la nouvelle : « Le tramway de Neuilly venait de passer la porte Maillot et il filait maintenant tout le long de la grande avenue qui aboutit à la Seine. » (Maupassant, 1974 [1881]: 193.) Cette ligne, qui est celle du trajet effectué par le tramway, est horizontale. Elle lie deux pôles : d'un côté, ce Paris où M. Caravan passe ses journées au travail et, de l'autre, son foyer. Son trajet ne changeant jamais, se reproduisant toujours de manière identique — le tramway part d'un point aux mêmes heures et va vers un autre point, pour finalement revenir, inlassable, à son lieu initial —, la ligne est ici mouvement continu et perpétuel de va-et-vient 1 . Elle laisse en cela remarquer une caractéristique propre à I'horizontalité sur laquelle nous reviendrons lorsqu'il sera question de M. Caravan : la reproduction immuable du même, du similaire.

Mentionnons d'ailleurs combien l'ensemble du texte se calque sur le modèle de ce trajet de tramway, sur son mouvement de va-et-vient : ainsi, le début de la nouvelle s'occupe de décrire la situation professionnelle de M. Caravan, sa place au ministère de la Marine, ses décorations; le cœur se consacre à l'événement familial et tient place au foyer; la dernière plainte de Caravan nous ramène d'un coup au bureau, à ce lieu initial d'où partait le personnage. Cependant, En famille ne se construit pas exclusivement sur la seule ligne droite du tramway; aussi, si l'horizontalité est la première à surgir dans le texte, la verticalité, sous forme de structure hiérarchique avec des « supérieurs », ne tarde pas à faire son apparition quelques lignes plus loin :

Il était vieux, maintenant, n'ayant point senti passer sa vie, car le collège, sans transition, avait été continué par le bureau, et les pions, devant qui il tremblait autrefois, étaient aujourd'hui remplacés par les 
chefs, qu'il redoutait effroyablement. Le seuil de ces despotes en chambre le faisait frémir des pieds à la tête; et de cette continuelle épouvante il gardait une manière gauche de se présenter, une attitude humble et une sorte de bégaiement nerveux. (195.)

La première ligne verticale, symbolique mais non moins réelle que le trajet du tramway², est celle de la structure hiérarchique du ministère de la Marine. Au sommet de cette structure, on trouve le chef; en bas, les subordonnés - dont fait partie, contre son gré, M. Caravan. II y a dans cette verticale, bien évidemment, domination d'un pôle sur l'autre : remarquons notamment la peur systématique qu'entretient M. Caravan à l'égard de ses chefs, qui le font «frémir des pieds à la tête »; qui le font « gagn[er] son bureau vivement, le cœur plein d'inquiétude, dans l'attente éternelle d'une réprimande pour quelque négligence qu'il aurait pu commettre » (194). Cependant, la peur n'est pas la seule émotion qui caractérise le rapport du personnage à ses supérieurs : existe également en lui un profond désir, celui d'en faire partie; les « employés de bureau », chez Maupassant, nourrissent « l'ordre qui les opprime, ou qui, en la personne des maîtres, les décore ou les épouse pour mieux les assujettir » (Peignot: 84) :

Rien n'était jamais venu modifier l'ordre monotone de son existence; car aucun événement ne le touchait en dehors des affaires du bureau, des avancements et des gratifications. Soit qu'il fût au ministère, soit qu'il fût dans sa famille (car il avait épousé, sans dot, la fille d'un collègue), il ne parlait jamais que du service. Jamais son esprit atrophié par la besogne abêtissante et quotidienne n'avait plus d'autres pensées, d'autres espoirs, d'autres rêves, que ceux relatifs à son ministère. (Maupassant: 194.)

Si l'horizontalité est associée dans la nouvelle à la reproduction immuable du même, la verticalité, mue par des rapports de domination, de peur et de désir, semble dans En famille être un moteur de changements, de transformations; c'est elle qui permet la différenciation et la valorisation. Monter sur la ligne, prendre un échelon, devenir un supérieur — ou au contraire, glisser vers le bas, perdre sa « place », déchoir —, la ligne verticale est ce qui donne véritablement de la texture aux choses.

Ouvrir l'espace, c'est d'abord le libérer d'un temps de succession et d'uniformisation. Nous ne cessons pas de revenir aux célèbres analyses de Jakobson qui définit justement le message poético-littéraire par son extension volumique : bi-axe, par suite de la projection de la métaphore sur la métonymie, ou de la similarité du versus dans la contiguïté du prorsus. Un vertical coupe l'horizontal. La «figure », un mixte de rhétorique et de spatialité, en résulte, qui ouvre et aère : elle donne sa place à la liberté dans la logique des néo-constructions. (Dagognet: 103.)

Un ensemble de mots se greffent à la verticalité dans le texte de Maupassant - notamment « avancement », « gratification », « décoration » ou « place ». Ces derniers montrent d'emblée ce que signifie l'accession, sur la 
ligne verticale, à des échelons supérieurs : un changement d'état, une évolution par rapport à l'état précédent. On n'est plus tout à fait le même lorsque l'on monte, on est un peu plus; il y a certainement dans l'ascension quelque chose qui relève d'une libération de ce « temps de succession et d'uniformisation » caractérisant toute la temporalité de la nouvelle. Une temporalité qui joue sur la même horizontalité que la ligne de tramway dans sa reproduction du même, et que l'on voit caractérisée par un tout autre ensemble sémantique : l'utilisation constante des adverbes « encore », « toujours », " invariablement » et « même »; les répétitions de comportements et de formules; les nombreux lieux communs; l'utilisation de noms et adjectifs référant à la routine et à l'habitude.

Ces deux lignes, l'une horizontale — le tramway —, l'autre verticale — la structure hiérarchique du ministère —, en cachent deux autres, plus fondamentales dans le texte : celle propre à l'arbre généalogique — le titre de la nouvelle y fait après tout directement référence - et celle sur laquelle s'inscrit $M$. Caravan, que nous pourrions qualifier de « ligne de vie ».

II est dit que M. Caravan « habitait, auprès du rond-point de Courbevoie, une petite maison à deux étages dont le rez-de-chaussée était occupé par un coiffeur » (197). La construction même de la maison, ici, s'inscrit dans une verticalité. Verticalité sur laquelle s'inscrivaient simultanément, lorsqu'il était question de la hiérarchie du ministère, peur et désir. Cette caractéristique singulière vient problématiser l'organisation spatiale de la maison de M. Caravan lorsqu'on apprend qu'« [a]u-dessus de lui, Caravan avait installé sa mère » (197). La mère constitue, effectivement, l'une des figures d'autorité. La chose est, à cet égard, assez explicite dans le texte. Ce n'est qu'à travers les admonestations et les colères que celle de M. Caravan nous est décrite :

Toujours de mauvaise humeur, elle ne passait point un jour sans querelles et sans colères furieuses. Elle apostrophait de sa fenêtre les voisins sur leurs portes, les marchandes des quatre-saisons, les balayeurs et les gamins qui, pour se venger, la suivaient de loin, quand elle sortait, en criant : — « À la chie-enlit! »(197.)

Lorsqu'elle s'adresse à son fils, toujours la mère demande-t-elle, ordonne-t-elle, exige-t-elle, impose-t-elle. Cela est entre autres présent dans le seul souvenir d'enfance que M. Caravan évoque d'elle, lorsqu'il cherche à se la rappeler vivante :

Et il revit soudain sa mère, autrefois, dans son enfance à lui, courbée à genoux devant leur porte, là-bas, en Picardie, et lavant au mince cours d'eau qui traversait le jardin le linge en tas à côté d'elle. II entendait son battoir dans le silence tranquille de la campagne, sa voix qui criait : « Alfred, apporte-moi du savon. »(205.)

Lorsqu'elle « revient à la vie », c'est encore avec un ordre que la mère s'adresse à son fils : «Toi, tu vas me 
remonter tout de suite ma commode et ma pendule. » (217.) Caravan semble entretenir la même relation avec sa mère qu'avec ses supérieurs du ministère, dans l'« attente éternelle d'une réprimande pour quelque négligence qu'il aurait pu commettre » (194). II n'est donc absolument pas fortuit que ce soit pour le ministère de la Marine - le « ministère de la mer », dit autrement — que travaille M. Caravan.

L'histoire des arbres généalogiques nous apprend que, de même que pour les structures hiérarchiques, des relations de pouvoir se jouent dans les représentations graphiques des familles. Pour les clercs du haut Moyen Âge, l'arbre généalogique sert à définir les degrés de parenté en matière d'héritage et de prohibition matrimoniale; il est particulièrement utile à la noblesse féodale, soucieuse qu'elle est de conserver ses titres de propriété et ses privilèges (Ingold: 139-140). C'est d'ailleurs la raison pour laquelle l'arbre généalogique est le plus souvent représenté de manière inversée : normalement, organiquement, les ancêtres devraient sur l'arbre être les racines, les descendants devraient être les branches. Or, lorsque l'enjeu était celui de la noblesse, les lignées étaient décrites comme des veines où coulait le sang dynastique : et cette " coulée " s'effectuait plus logiquement de haut en bas (Ingold: 140-141). De même que pour la structure hiérarchique, c'est le haut de l'arbre généalogique qui est le plus valorisé : plus près du ciel, plus près de Dieu, il est l'image de la pureté originelle, sans mélange (Ingold: 140-141).

Mais, comme pour la ligne verticale de la structure hiérarchique, M. Caravan désire-t-il autant sa mère qu'il la craint? II est intéressant, à cet égard, de voir qu'en tous points la femme de $\mathrm{M}$. Caravan correspond à la mère. Les deux sont appelées, indistinctement, Mme Caravan; le physique de l'une et de l'autre est décrit exactement de la même façon, dans leur sécheresse et leur maigreur extrêmes; toutes deux sont des figures dures et autoritaires. M. Caravan, à la maison, dit se sentir toujours pris entre sa mère et sa femme; il tente de plaire, en les ménageant et en accédant à toutes leurs demandes, indifféremment à sa femme comme à sa mère. L'ambigüité entretenue entre mère et femme, entre les rôles et fonctions qu'elles ne cessent de s'échanger dans la narration, inscrit ainsi également le désir dans la verticalité de l'arbre généalogique. Le corps de la mère est aussi le seul auquel s'intéressera M. Caravan dans le cadre de la narration :

II revoyait la « maman » plus jeune, vêtue de robes usées sur elle, portées si longtemps qu'elles semblaient inséparables de sa personne; il la retrouvait dans mille circonstances oubliées : avec des physionomies effacées, ses gestes, ses intonations, ses habitudes, ses manies, ses colères, les plis de sa figure, les mouvements de ses doigts maigres, toutes ses attitudes familières qu'elle n'aurait plus. (205.)

C'est donc vers le haut des deux lignes verticales, la structure hiérarchique du ministère et l'arbre généalogique, que tend $\mathrm{M}$. Caravan; vers les pôles occupés par les chefs d'une part, par la mère de l'autre. Cependant, dans les deux cas, il est impossible à M. Caravan de monter : le haut de la ligne, du début à la fin, 
reste complètement inaccessible. M. Caravan ne sera pas promu, quelqu'un d'autre que lui aura sa place. L'unique fois où il saura s'approprier ce qui relevait du pôle supérieur — la commode et la pendule de la mère - se soldera au final par un échec; ce qui aura été pris en haut devra, ultimement, retourner en haut; et M. Caravan devra, inévitablement, reprendre sa place en bas; cette place qui lui était et lui restera allouée. Sans être totalement au bas de la ligne verticale, on n'arrive plus, dans En famille, à en gravir les échelons; on est coincé dans une sorte d'entre-deux d'où il n'est pas possible de se dégager.

L'impression d'être coincé au milieu de la ligne est évoquée plusieurs fois au sein de la nouvelle : M. Caravan n'habite ni en ville, ni à la campagne, mais dans cette banlieue décrite comme une « campagne à dépotoirs qui borde Paris » (193), « remplie d'une population de province » (203); il est décoré, récompensé pour ses trente années de service, rempli d'orgueil et parle de sa croix à tout propos mais se fait dépasser par tous les nouveaux venus; M. Caravan habite le premier étage d'une maison qui en a deux. D'ailleurs, nous remarquons que la nouvelle elle-même est écrite dans une espèce d'entre-deux; qu'elle représente comme une fissure dans l'atemporalité de la bureaucratie ministérielle (ce « temps de succession et d'uniformisation ») vécue par M. Caravan - fissure qui se referme presque aussitôt entrouverte.

Aussi pouvons-nous affirmer que si M. Caravan évolue sur une ligne, cette dernière n'est certainement pas verticale. Coincé quelque part au milieu de la verticalité, il n'apparaît jamais être en mesure d'atteindre le haut. C'est donc sur une ligne horizontale que M. Caravan évolue - pareille au trajet du tramway, sa vie est celle d'une reproduction exacte et immuable du même. L'humanisation du tramway, en début de nouvelle, n'est donc pas anodine. " La petite machine, attelée à son wagon, cornait pour écarter les obstacles, crachait sa vapeur, haletait comme une personne essoufflée qui court; et ses pistons faisaient un bruit précipité de jambes de fer en mouvement » (193) : cette description est à rapprocher de ce M. Caravan qui nous est peint dans En famille — de ce M. Caravan qui sans répit, et toujours aux mêmes heures, passe du ministère à la maison, dans un perpétuel mouvement maladroit qui ne réussit pas à porter fruit $\underline{3}$. S'il faut rapprocher la mère du « ministère de la mer », peut-être faut-il également souligner la similitude qui existe entre le nom de Caravan et celui d'un moyen de transport.

Convoitant le haut de la ligne, M. Caravan est confiné à l'horizontalité. II est, à cet égard, un peu comme ce tramway qui, de manière routinière et indépassable, se déplace entre Paris et la banlieue. Mais dans la nouvelle, un autre objet central s'apparente au trajet, à la ligne propre de M. Caravan : la pendule qu'il descend de l'appartement de sa mère. Cette horloge est un symbole lourd de sens : elle renvoie d'abord à la monotonie du temps qui, sans changement, s'écoule toujours également pour notre personnage principal. Elle est également un rappel du temps bureaucratique, ce « temps lié à l'asservissement de l'homme qui va à la mort » (Cury: 92) : le ministère étant apparenté dans En famille à une administration militarisée, les 
fonctionnaires étant comparés à des prisonniers et à des forçats rivés au carton vert, il est très difficile dans cette situation de ne pas songer à toute la vérité qui s'exprime dans la locution « faire son temps ». Et finalement, du simple point de vue du mouvement, tout comme M. Caravan, le pendule de la pendule tend constamment vers le haut, mais toujours revient-il, inlassablement, au même point. M. Caravan est, dans En famille, l'homme-pendule.

\section{Caravan, l'otage de la lettre}

Penchons-nous maintenant sur une autre propriété de la ligne dans le texte de Maupassant : sa propension à contaminer les pôles qu'elle lie. La ligne verticale liant l'étage de la famille Caravan à celui de la mère décédée joint également, d'une manière symbolique, autre chose : la vie, qui se trouve à l'extrémité inférieure, à la mort, située à l'extrémité supérieure. D'un côté, nous avons la famille, les repas, les enfants; de l'autre côté, le cadavre de la mère, sur lequel « des petites mouches grimpaient, allaient, venaient, se promenaient sans cesse, visitaient la vieille, attendant leur heure prochaine » (109). Cependant s'effectue, dans En famille, un drôle de renversement : la mère s'avère à la fin être bel et bien en vie, alors que M. Caravan, lui — qui devrait se situer du côté des vivants - possède dans sa reproduction constante et perpétuelle de la même journée des points communs incontestables avec la mort. II semble que la ligne, en joignant ensemble deux pôles, ne les oppose pas tout à fait entre eux; qu'au contraire, cette ligne possède des propriétés contaminatrices. Les points qui se trouvent unis entre eux (la mort et la vie, le travail et la famille, le rite de mort et la bureaucratie d'un ministère), malgré leur apparente dichotomie, forment des mélanges pas toujours orthodoxes.

De la même manière que pour celle existant entre les Caravan et la mère morte, la ligne horizontale du tramway ne fait pas que lier Paris à la banlieue : elle joint également deux mondes, celui de la littératie — de l'écriture et du calcul — à celui de l'oralité. Paris, et le ministère en particulier, sont des lieux où règnent l'ordre, la structure hiérarchique et le travail du papier : ces endroits correspondent à des « administrations militarisées », où travaillent des «forçats rivés au carton vert » (Maupassant: 195); ils sont ce qui force M. Caravan à discipliner son corps (Foucault, 1993 [1975]), à se raser tous les matins, à écurer ses ongles avec plus de soin, à « chang[er] de linge tous les deux jours par un légitime sentiment de convenances et de respect pour l'Ordre national dont il faisait partie » (195). De l'autre côté de la ligne, la maison des Caravan se situe, elle, dans ce qu'on n'arriverait pas à écrire sur des papiers administratifs; elle s'inscrit dans le rituel, dans le corps - mais également, au contraire du ministère, dans le désordre :

On se dirigea vers la chambre mortuaire. Les quatre femmes entrèrent à pas de loup, aspergèrent le drap l'une après l'autre avec l'eau salée, s'agenouillèrent, firent le signe de la croix en marmottant une prière, puis, s'étant relevées, les yeux agrandis, la bouche entrouverte, considérèrent longuement le cadavre, pendant que la belle-fille de la morte, un mouchoir sur la figure, simulait un hoquet désespéré. 
Quand elle se retourna pour sortir, elle aperçut, debout près de la porte, Marie-Louise et Philippe-

Auguste, tous deux en chemise, qui regardaient curieusement. Alors, oubliant son chagrin de commande, elle se précipita sur eux, la main levée, en criant d’une voix rageuse : « Voulez-vous bien filer, bougres de polissons! »

Étant remontée dix minutes plus tard avec une fournée d'autres voisines, après avoir de nouveau secoué le buis sur sa belle-mère, prié, larmoyé, accompli tous ses devoirs, elle retrouva ses deux enfants revenus ensemble derrière elle. Elle les talocha encore par conscience; mais, la fois suivante, elle n'y prit plus garde; et, à chaque retour de visiteurs, les deux mioches suivaient toujours, s'agenouillant aussi dans un coin et répétant invariablement tout ce qu'ils voyaient faire à leur mère. (212.)

Il faut d'abord remarquer que le rituel de mort n'appartient pas au domaine du papier, de l'administration ou de la littératie. II appartient au domaine de l'oralité, au domaine du corps. On entre en silence, on s'agenouille; on asperge le cadavre d'eau bénite, on fait des signes de croix; on pleure. C'est d'ailleurs par la reproduction corporelle des différents gestes effectués par la mère que ce rituel se transmet aux enfants, et que MarieLouise arrive à le répéter en « recommanç[ant] chaque fois les simagrées maternelles avec une perfection absolue » (213). Mais dans ce même extrait, il n'y a pas que l'oralité du foyer qui s'oppose à la culture écrite de Paris. Dans tout le déroulement du rituel, il existe un désordre qui ne serait sans doute pas permis dans un ministère. Que soit utilisée de l'eau salée pour remplacer l'eau bénite; qu'on oublie « son chagrin de commande " pour lancer des insultes aux enfants; qu'on ne prenne plus garde à ceux-ci qui observent avec curiosité ce qui devrait être un spectacle larmoyant : tout cela correspond à un dévoiement certain du rite de mort, mais fait tout de même bien partie de l'oralité et du corps qui caractérisent le foyer des Caravan.

Cette maison, par rapport à la rigidité de la structure ministérielle, ne constitue que désordre : ainsi, nous l'avons vu, la mère de M. Caravan gronde-t-elle contre tout ce qui bouge; les enfants « galopin[ent] dans les ruisseaux de l'avenue avec tous les polissons du quartier " (197); soulignons également que c'est l'absence de papier — l'absence de testament — qui fait le plus désordre dans En famille. Ce désordre va jusqu'à s'inscrire dans le corps même de Mme Caravan :

Elle n'avait jamais été jolie; elle était laide maintenant, de petite taille et maigrelette. L'inhabileté de sa vêture avait toujours fait disparaître ses faibles attributs féminins qui auraient dû saillir avec art sous un habillage bien entendu. Ses jupes semblaient sans cesse tournées d'un côté; et elle se grattait souvent, n'importe où, avec indifférence du public, par une sorte de manie qui touchait au tic. Le seul ornement qu'elle se permît consistait en une profusion de rubans de soie entremêlés sur les bonnets prétentieux qu'elle avait coutume de porter chez elle. (198.)

C'est dans l'allure même de l'épouse Caravan que se manifeste le désordre : le texte souligne « [l]'inhabilité de 
sa vêture " par rapport à ce qui aurait dû être (soit - dit autrement - ce déplacement qui s'opère dans la maison Caravan de l'ordre au désordre); Mme Caravan se gratte « avec indifférence du public »; son bonnet ne tient jamais en place, il est « sans cesse chaviré sur une oreille » (198). La maison Caravan se trouve du côté du désordre, de l'oralité et du corps; à l'opposé, du côté du ministère, règnent l'ordre, l'administration, la bureaucratie, le papier, l'écrit — la raison graphique.

Cependant, la ligne droite dans En famille, qu'elle soit verticale ou horizontale, ne sépare pas hermétiquement les pôles qu'elle lie; comme nous l'avons souligné, elle possède des propriétés contaminatrices. Ainsi, les extrémités échangent entre elles des caractéristiques qui appartiennent à l'une ou à l'autre. Si M. Caravan, qui travaille comme commis pour le ministère de la Marine, devrait appartenir davantage au papier qu'au corps, c'est pourtant ce dernier qui est constamment mis de l'avant dans le texte; et Mme Caravan, qui s'occupe du rituel de mort, devrait normalement se situer plus du côté de l'oralité - cependant, c'est elle la vraie bureaucrate de la famille.

Dès l'incipit, c'est aux hommes que devrait principalement revenir la raison graphique : ainsi parle-t-on « des messieurs las du bureau, [...] une épaule un peu remontée par les longs travaux courbés sur les tables » (193). Dans la même phrase sont mises en parallèle les femmes, dont on semble prévenir d'avance qu'elles se trouvent davantage situées du côté du corps : ce sont « de grosses dames aux toilettes farces, de ces bourgeoises de banlieue qui remplacent la distinction dont elles manquent par une dignité intempestive » (193). Mais c'est dans le dévoiement de cette dynamique anticipée que les propriétés contaminatrices de la ligne droite viennent ici opérer. Si elle prend en charge le rituel de mort, si c'est elle qui secoue « le buis sur sa belle-mère », Mme Caravan est également celle qui se soucie le plus du papier : d'ailleurs dit-on que tous les soirs, à table et au lit, elle cause avec son mari « longuement des affaires du bureau, et, bien qu'elle eût vingt ans de moins que lui, il se confiait à elle comme à un directeur de conscience, et suivait en tout ses conseils » (197). Mme Caravan manie l'Annuaire de la marine pour savoir par qui son mari s'est fait dépasser au ministère (198); c'est elle qui pense au testament, à la déclaration à la mairie, aux commandes, à l'imprimerie, au notaire, au télégraphe; elle qui dresse la fameuse liste des choses que doit faire M. Caravan le lendemain du décès de sa mère, «nomenclature dont [lui-même est] épouvanté » (211). Mme Caravan maîtrise également le calcul :

Lorsqu'ils furent chez eux, elle poussa un grand soupir. " Le plus gros est fait, dit-elle; allons chercher le reste. 》

Mais les tiroirs du meuble étaient tout pleins des hardes de la vieille. II fallait bien cacher cela quelque part.

Mme Caravan eut une idée : « Va donc prendre le coffre à bois en sapin qui est dans le vestibule; il ne 
vaut pas quarante sous, on peut bien le mettre ici. » Et quand le coffre fut arrivé, on commença le transport. (210.)

Ainsi est-elle capable de mesurer, avec les prix et les valeurs précises en tête, le profit qu'elle fera en échangeant la commode de la mère pour leur coffre en bois. Ce n'est certainement pas un hasard si, en nous la présentant, la narration maupassantienne précise le lien qui rattache l'épouse au ministère pour lequel travaille son mari : Mme Caravan est « la fille d’un collègue » (194) qu'il a épousée sans dot.

Que M. Caravan l'ait mariée sans dot nous donne un autre indice. Si cette dernière est calculatrice, son mari, lui, ne sait pas compter. D'ailleurs, quoique son métier de commis devrait le rapprocher de la raison graphique, c'est pourtant son corps qui est constamment ramené au premier plan de la nouvelle. M. Caravan est décrit comme « un homme petit et gros, la figure bouffie, le ventre tombant entre ses jambes ouvertes » (193-194); plus loin, le docteur Chenet constate « la figure rougeaude de son voisin, son cou graisseux, son bedon tombant entre deux jambes flasques et grasses, toute sa rondeur apoplectique de vieil employé ramolli ", allant jusqu'à dire de lui qu'il n'est qu'un « plein-de-soupe » (196). Et lorsque sa mère meurt, plus rien n'existe d'autre de M. Caravan que sa personne effondrée : «Caravan s'abattit sur le corps, secoué par des sanglots précipités; et il baisait convulsivement la figure rigide de sa mère en pleurant avec tant d'abondance que de grosses larmes tombaient comme des gouttes d'eau sur le visage de la morte » (200-201). Plus loin : « [i]l larmoyait comme un gros enfant, avec des hoquets convulsifs, avachi, les bras pendants, les jambes molles; et il descendit l'escalier sans savoir ce qu'il faisait, remuant les pieds machinalement » (202). Finalement, suite à sa promenade avec le docteur : «[S]e cramponnant au docteur, il poussa des gémissements. Ses jambes flasques tremblaient; toute sa grosse personne était secouée par les sanglots et il balbutiait : "Ma mère, ma pauvre mère, ma pauvre mère!..." » (206.) Bien que M. Caravan travaille pour ce qu'il appelle “ l'Ordre national » et que Mme Caravan prenne en charge le rituel entourant la mort de sa belle-mère, nous voyons très bien ici qu'oralité et raison graphique n'appartiennent pas qu'à un côté ou à un autre; qu'elles communiquent entre eux, qu'elles se contaminent.

Il faut remarquer finalement combien la ligne droite, même horizontale, n'instaure jamais dansEn famille des rapports d'égalité entre les différents pôles qu'elle unit. Ainsi, Paris est clairement représenté dans la nouvelle comme plus élevé, hiérarchiquement, que la banlieue où habite la famille Caravan. Et dans le rapport entre oralité et culture écrite, la supériorité de la dernière sur la première est explicite. Cette domination de la lettre sur le corps est annoncée dans les premières phrases de la nouvelle : on dit bien que les messieurs sont « las du bureau, la figure jaunie, la taille tournée, une épaule un peu remontée par les longs travaux courbés sur les corps » (193). Ce corps, dans En famille, on se trouve obligé de l'ordonner pour travailler au service de la lettre : M. Caravan « supprim[e] les pantalons de couleur et les vestons de fantaisie » (195), se rase tous les 
matins, écure ses ongles avec plus de soin, change de linge tous les deux jours par, rappelons-le, « un légitime sentiment de convenances et de respect pour l'Ordre » (195). La lettre ordonne et domine le corps; elle fait de celui qui doit la manier son otage (Gardey, 2001). Ce n'est pas pour rien que sont comparées dans le texte les « administrations militarisées » à une « longue et misérable servitude » (185); que les travailleurs y sont assimilés à de " tristes forçats rivés au carton vert » (185); enfin, que M. Caravan « entr[e] au ministère à la façon d'un coupable qui se constitue prisonnier » (194). L'écriture, historiquement, correspond bel et bien à un outil de domination, comme l'explique Jack Goody :

La littératie a conféré un grand pouvoir au clergé, presque entièrement masculin, ainsi qu'à l'élite (souvent exclusivement masculine) qu'il formait. En conséquence, la minorité des lettrés était investie d'un pouvoir sur la majorité des illettrés (dont une grande proportion de femmes), qui n'avaient qu'un accès indirect au texte canonique. Avec certaines exceptions, cette situation perdura dans le domaine religieux pendant presque cinq mille ans, depuis l'invention de l'écriture, en passant par la diffusion des écoles après la Renaissance, jusqu'au XIX ${ }^{e}$ siècle, où l'instruction universelle commença à devenir une réalité dans quelques-unes des principales puissances mondiales. (2007: 189-190.)

Si la mort de la mère de M. Caravan n'advient pas dans le texte, donc, c'est peut-être surtout pour la raison qu'elle n'a jamais pu être officialisée par le papier - ce papier qui domine le corps. Le rituel de mort n'a aucune conséquence dans En famille, parce que personne n'a encore pris le temps de la consigner par écrit. Le problème aurait sans aucun doute été tout autre si tel avait été le cas : évoquons à cet égard le Colonel Chabert, ce vivant que le papier avait cependant déclaré mort (Balzac, 1994 [1832]). Effectivement, « dans certaines situations rituelles, l'écriture établit un conformisme, une orthodoxie, comme dans les religions du Livre, qui perdurent inchangés de siècle en siècle » (Goody, 2007: 71). Une fois posée sur le papier, une situation ne peut plus être annulée aussi facilement que lorsqu'elle n'y figure pas - un autre aspect d'une domination de l'écriture sur l'oralité. «Qu'est-ce que je vais dire à mon chef? » (218), laisse glisser M. Caravan à la toute fin de la nouvelle. Nous voyons bien ici que la seule chose qui sera comptabilisée au final, ce ne sera pas la mort de sa mère. Ce sera plutôt cette journée au service de la lettre que M. Caravan, bien malgré lui, aura perdue.

Nous avons vu au cours de cette analyse la manière dont la ligne et la lettre permettent de mieux lire les tensions qui sont en jeu dans la nouvelle de Maupassant. Effectivement, comprendre les organisations linéaires horizontale et verticale d'En famille; mettre en parallèle ces organisations avec les rapports complexes qu'entretiennent les personnages avec la littératie : tout cela semble exposer une interprétation riche que l'auteur fait de son époque. L'entre-deux dans lequel se situe M. Caravan au sein de son ministère, 
où il cherche à " gagner sa place ", où tous ses collègues deviennent de facto ses compétiteurs et où le temps passé à un poste n'a plus aucune valeur, c'est aussi celui que fournit le cadre de la seconde révolution industrielle - au cœur de laquelle se joue l'action de la nouvelle (l'importance qu'y prend le tramway n'est, à ce titre, certainement pas une coïncidence). Sans faire de Maupassant un « socialiste » (Peignot: 86) dont les nouvelles possèdent « le pouvoir détonnant d'un pamphlet » (Peignot: 88), il est en effet possible de comprendre En famille comme le soubresaut, la dernière tentative de personnages de faire comme si le « temps d'avant » continuait à avoir un sens - une efficacité — dans un monde qui change avec vélocité. Mais le factice du rite de mort mis en scène par la nouvelle, comparé à la réalité matérielle d'un emploi qu'on dit se rapprocher des travaux forcés accomplis dans les bagnes, laisse percevoir contre ces intentions un tableau sans équivoque : les temps, de même que les règles du jeu, ont bel et bien changé.

1. Sur l'histoire des tramways à Paris, on se reportera à l'étude de Jean Robert (1959).

2. Concernant l'aspect performatif des lignes symboliques, voir Tim Ingold (70).

3. Bernard Demont a analysé ces enjeux dans une autre nouvelle de Maupassant analogue sous certains aspects (2005 [1952]: $75-$ 77).

\section{Bibliographie}

BALZAC (DE), Honoré. 1994 [1832]. Le Colonel Chabert, introduction, notes, commentaire et dossier de Stéphane Vachon. Paris : Librairie générale française, «Classiques de poche », $191 \mathrm{p}$.

CuRY, Maurice. 1969. «La notion du temps dans la nouvelle». Europe, vol. 47, no 482, juin, p. 88-92. Dagognet, François. 1973. Écriture et iconographie. Paris : Vrin, « Problèmes \& Controverses », 172 p. Demont, Bernard. 2005 [1952]. Représentations spatiales et narration dans les contes et nouvelles de Guy de Maupassant. Une rhétorique de l'espace géographique. Paris : Honoré Champion, 540 p. Foucault, Michel. 1993 [1975]. Surveiller et punir. Paris : Gallimard, « Tel », 400 p. GARDEY, Delphine. 2001. La dactylographe et l'expéditionnaire. Histoire des employés de bureau : 18901930. Paris : Belin, $335 \mathrm{p}$.

Goody, Jack. 1979. La raison graphique. La domestication de la pensée sauvage. Paris : Minuit, 274 p. Goody, Jack. 2007. Pouvoirs et savoirs de l'écrit, traduit par Claire Maniez, coordination par Jean-Marie Privat. Paris : La Dispute, 269 p.

INGOLD, Tim. 2011. Une brève histoire des lignes, traduit par Sophie Renaut. Bruxelles : Zones sensibles, $256 \mathrm{p}$.

Maupassant (DE), Guy. 1974 [1881]. «En famille », dans Contes et nouvelles, édition de Louis Forestier, préface d'Armand Lanoux. Paris : Gallimard, «Bibliothèque de la Pléiade », t. 1, p. 193-218. 
PEIGNOT, Jérôme. 1969. «Les employés de bureau ». Europe, vol. 47, no 482, juin, p. 84-88.

RoberT, Jean. 1959. Les tramways parisiens. Paris : G. Fuseau, 255 p. 